\title{
New COMPASS Results on Transverse Spin Asymmetries in Hadron Pair Production in DIS
}

\author{
G. Sbrizzai \\ on behalf of the COMPASS Collaboration \\ Physics Deparement, Trieste University, Via Valerio 2, Trieste, 34127, Italy \\ Giulio.Sbrizzai@ts.infn.it
}

Published 29 February 2016

\begin{abstract}
The COMPASS measurements of the azimuthal asymmetries in the hadron pairs production in DIS on transversely polarised targets are here presented. Results on oppositely charged pion and kaon pairs produced on both deuteron and proton targets are shown. They have been used to update the calculation of the Transversity function which was previously extracted from the asymmetries measured from un-identified hadron pairs. Also a new method for the direct measurement of the integral of Transversity using both COMPASS and BELLE results is presented.
\end{abstract}

Keywords: Nucleon spin structure; transversity; COMPASS; BELLE.

PACS numbers: 13.60.-r, 13.88.+e, 14.20.-c, 14.65.-q

\section{Theoretical Framework and Data Selection}

The transverse spin distribution $h_{1}^{q}$, often referred to as Transversity and which gives the probability difference of finding a quark $q$ polarised parallel or antiparallel to the spin of a transversely polarised nucleon, is one of the most interesting quantities which describe the spin structure of the quark inside the nucleon. Transversity can be assessed in SIDIS both in single hadron production $l N \rightarrow l^{\prime} h X$, by measuring the Collins asymmetries, and in di-hadron production $l N \rightarrow l^{\prime} h_{1} h_{2} X$. In this case the amplitudes of the asymmetries on the azimuthal angle $\phi_{R S}=\phi_{R}+\phi_{S}-\pi$ are measured, where $\phi_{R}$ is the azimuthal angle of the relative transverse momentum of the hadron pair $\boldsymbol{R}$ and $\phi_{S}$ is the azimuthal angle of the spin of the initial quark, both calculated with respect to the scattering plane in the $\gamma^{*}-N$ system. A detailed description of the process can be found in Ref. 1. The amplitude of the asymmetry is calculated in a collinear framework and is given by the product of

This is an Open Access article published by World Scientific Publishing Company. It is distributed under the terms of the Creative Commons Attribution 3.0 (CC-BY) License. Further distribution of this work is permitted, provided the original work is properly cited. 
transversity and the interference fragmentation function, which describes the spindependent part of the fragmentation of a transversely polarised quark into a pair of unpolarised hadrons ${ }^{2-3}$ after integration over the azimuthal angle of the total transverse momentum of the pair. These asymmetries are measured at COMPASS using a positive muon beam of $160 \mathrm{GeV} / \mathrm{c}$ after standard DIS events selection, namely asking the four-momentum transfer $Q^{2}>1(\mathrm{GeV} / c)^{2}$, the fractional energy transfer of the muon $0.1<y<0.9$ and the hadronic invariant mass $W>5 \mathrm{GeV} / c^{2}$. All possible combinations of oppositely charged hadron-pairs originating from the vertex are taken into account in the analysis. Each of these hadrons has to have a fractional energy $z>0.1$ and a Feynman $x_{F}>0.1$, to ensure that the hadrons are not produced in target fragmentation. Exclusively produced $\rho$ mesons are rejected by a cut on the missing energy $E_{\text {miss }}>3 \mathrm{GeV}$. Finally a cut of $R_{T}>0.07 \mathrm{GeV} / c$ ensures a well defined azimuthal angle $\phi_{R}$.

\section{Results from the 2003-04 Deuteron data}

The di-hadron asymmetries $A_{d}^{2 \pi}$ of pion-pairs $\pi^{+} \pi^{-}$for the data collected in 20032004 on the deuteron target are shown in Fig. 1. They are small and compatible with zero within the uncertainties and no specific trend is visible for their dependence on $x, z$ and the invariant mass of the di-hadron system $M_{i n v} \cdot{ }^{4}$ This result, in line with previous measurements ${ }^{6-}, 5$ is interpreted as the consequence of a cancellation of the $u$ and $d$ quark contributions to the total Transversity distribution of the deuteron. ${ }^{7-8}$ The asymmetries of the other identified pair combinations $K^{+} K^{-}$,

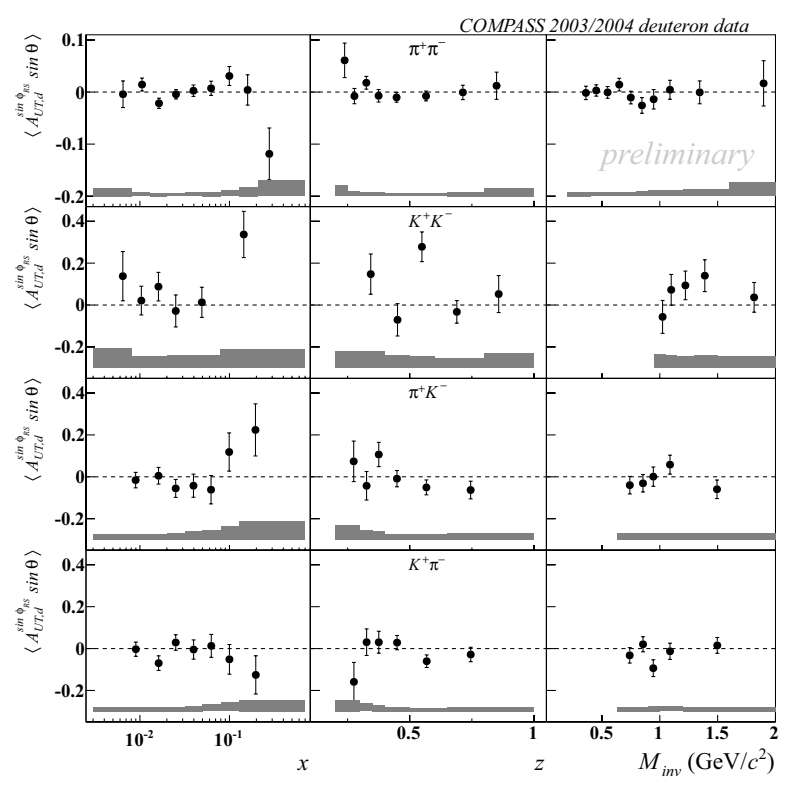

Fig. 1. The identified di-hadron asymmetries rom the 2003-04 deuteron data. 
$\pi^{+} K^{-}$and $K^{+} \pi^{-}$are compatible with zero within their uncertainties or give only weak hints of nonzero signals.

\section{Results from the Combined 2007/2010 Proton Data}

COMPASS has measured the di-hadron asymmetry on a proton target $\left(\mathrm{NH}_{3}\right)$ in 2007 and in 2010, and the combined results for $h^{+} h^{-}$pairs as a function of $x, z$ and $M_{i n v}$ are published in Ref. 9. Results on identified hadrons are available and they are shown in Fig. 2. The pion-pair asymmetries $A_{p}^{2 \pi}$ exhibit a strong signal in $x$, there is no clear trend in the $z$ and some structure can be seen in the asymmetries as function of $M_{i n v}$, in particular at the $\rho$ mass. The asymmetries of the other identified pair combinations $K^{+} K^{-}$and $K^{+} \pi^{-}$give some weak indications of nonzero signals as functions of their invariant masses.

\section{Extraction of Transversity}

The complete set of di-hadron asymmetries obtained from the COMPASS data taken on both transversely polarized deuteron and proton targets gives the unique possibility to extract the Transversity distributions $x h_{1}^{q}(x)$ of $u$ and $d$ valence quarks separately. This was first done in Ref. 11, using the COMPASS unidentified hadron data and following an original procedure ${ }^{10}$ which used only the proton data from COMPASS, only from the 2007 run, and HERMES. Later the procedure was extended to include also the COMPASS deuteron data. ${ }^{12}$ Now the

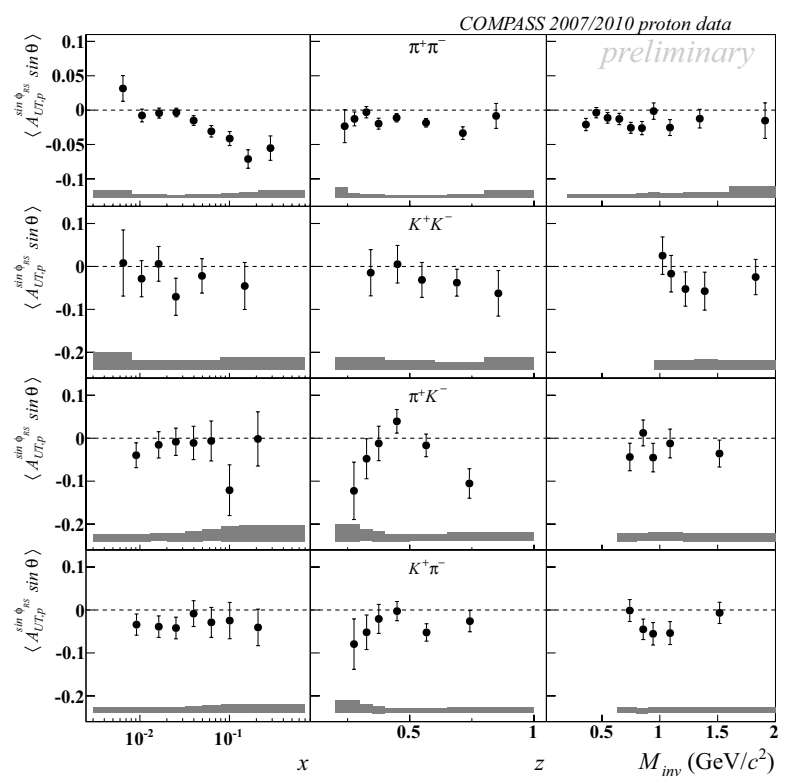

Fig. 2. The identified di-hadron asymmetries from the combined 2007/2010 proton data. 

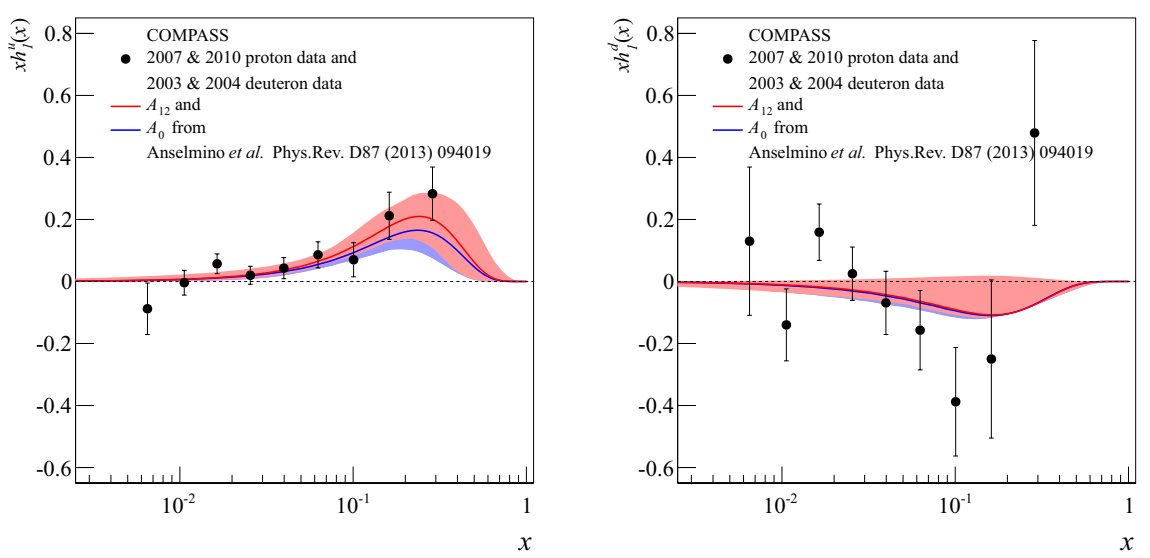

Fig. 3. Point-by-point Transversity distributions for $u$ (left) and $d$ (right) valence quarks obtained from this data. They are compared to the corresponding results extracted from the single hadron Collins asymmetries.

extraction of $x h_{1}^{u}(x)$ and $x h_{1}^{d}(x)$ has been updated using the pion-pairs asymmetries measured on the polarized proton target from the 2010 data. ${ }^{4}$ The starting points are the linear combinations $x h_{1, p}\left(x, Q^{2}\right)=x h_{1}^{u}\left(x, Q^{2}\right)-\frac{1}{4} x h_{1}^{d}\left(x, Q^{2}\right)$ and $x h_{1, d}\left(x, Q^{2}\right)=x h_{1}^{u}\left(x, Q^{2}\right)+x h_{1}^{d}\left(x, Q^{2}\right)$ extracted in Ref. 12 . The bin-by-bin ratios between $x h_{1, p / d}$ and the COMPASS di-hadron asymmetries of $h^{+} h^{-}$pairs used as an input in the extraction are then calculated. These ratios contain the information on the unpolarized process as well as on the interference fragmentation function. They are used as conversion factors in order to calculate the point-by-point amplitudes of the Transversity distribution $x h_{1, p}$ with the new $\pi^{+} \pi^{-}$results. The final $u$ and $d$ valence quark Transversity distributions are obtained by solving the system of two linear equations. Figure 3 shows the results for $x h_{1}^{u}$ and $x h_{1}^{d}$. A non zero trend at large $x$ values is evident in both distributions with opposite sign for the $u$ and $d$ quarks and the strength of the signal is compatible within the uncertainties. The $x h_{1}^{u}$ and $x h_{1}^{d}$ distributions of Fig. 3 are also well compatible with the extraction of Ref. 13 carried out using single hadron Collins asymmetries and BELLE $e^{+} e^{-}$ results on Collins fragmentation function.

\section{A New Method for a Measurement of the Integral of Transversity}

An interesting quantity can be calculated by combining the COMPASS asymmetries $A_{p}^{2 \pi}$ extracted as function of $z$ or $M_{i n v}$ with the measurements performed by BELLE ${ }^{14}$ on the interference fragmentation function (IFF) from the $e^{+} e^{-} \rightarrow\left(\pi^{+} \pi^{-}\right)\left(\pi^{+} \pi^{-}\right)$reaction. The di-hadron asymmetry measured on the 

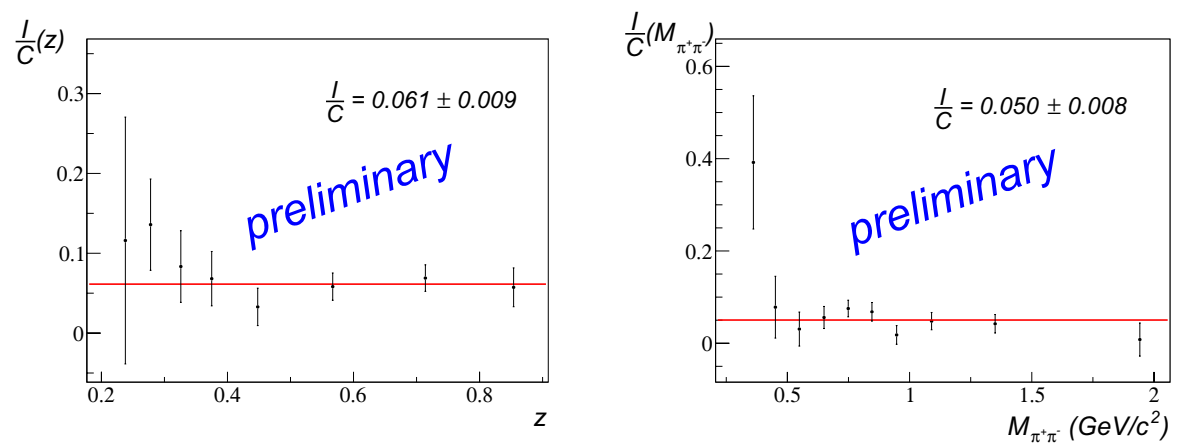

Fig. 4. Points calculated for $\frac{I}{C}(z)$ (left) and $\frac{I}{C}\left(M_{i n v}\right)$ (right). The average value of $\frac{I}{C}$ is also shown.

transversely polarized proton target in bins of $z$ can be written as

$$
A_{p}^{2 \pi}(z)=\frac{I}{C} \cdot \beta(z),
$$

where

$$
\begin{gathered}
I=\frac{2 \pi s \alpha^{2}}{\left\langle D_{N N}\right\rangle} \cdot \int_{\omega_{x}, \omega_{y}} d x d y \frac{2(1-y)}{Q^{4}}\left[4 x h_{1}^{u-\bar{u}}(x)-x h_{1}^{d-\bar{d}}(x)\right], \\
C=2 \pi s \alpha^{2} \cdot \int_{\omega_{x}, \omega_{y}} d x d y \frac{1+(1-y)^{2}}{Q^{4}}\left[4 x f_{1}^{u+\bar{u}}(x)+x f_{1}^{d+\bar{d}}(x)\right],
\end{gathered}
$$

being $D_{N N}=\frac{2(1-y)}{1+(1-y)^{2}}$ and $f_{1} \cdots$ are the unpolarized PDFs. The usual assumptions on the fragmentation functions (FFs) are made ${ }^{15}$ and the $Q^{2}$ dependence of the FFs and parton distribution functions (PDFs) is neglected. The term $\beta(z)$ contains only the favored IFF $H_{f}^{2 h}$ and the unpolarized di-hadron $\mathrm{FF} D_{f}^{2 h}$

$$
\beta(z)=\frac{\int_{\omega_{M_{i n v}}} H_{f}^{2 h}\left(z, M_{i n v}\right) d M_{i n v}}{\int_{\omega_{M_{i n v}}} D_{f}^{2 h}\left(z, M_{i n v}\right) d M_{i n v}} .
$$

In the same way the asymmetry measured in bins of $M_{i n v}$ can be written as

$$
A_{p}^{2 \pi}\left(M_{i n v}\right)=\frac{I}{C} \cdot \beta^{\prime}\left(M_{i n v}\right),
$$

where

$$
\beta^{\prime}\left(M_{i n v}\right)=\frac{\int_{\omega_{z}} H_{f}^{2 h}\left(z, M_{i n v}\right) d z}{\int_{\omega_{z}} D_{f}^{2 h}\left(z, M_{i n v}\right) d z} .
$$

The terms defined in Eq. (4) and (6) can be calculated from the BELLE azimuthal asymmetries $a_{12}\left(z, M_{i n v}\right)$ measured in bins of $z$ and $M_{i n v}$ and given in Ref. 14, since the ranges of $z$ and $M_{i n v}$ are similar to the ones covered by COMPASS. Indeed, using the relations among FFs of Refs. $15-14$, it can be written that $\beta(z)=$ 
$\frac{1}{\sqrt{k}} \cdot\left\langle a_{12}(z)\right\rangle_{M_{i n v}}$ where $\left\langle a_{12}(z)\right\rangle_{M_{i n v}}$ is the weighted mean calculated over the bins in $M_{i n v}$ and $k=\frac{-5}{8} \frac{s^{2}}{1+c^{2}} \cdot a_{I}$. The angular quantities $\frac{s^{2}}{1+c^{2}}$, defined in Ref. 14, are almost independent from $z$ and $M_{i n v}$ and $a_{I}$ is proportional to the asymmetries integrated over the whole $z, M_{\text {inv }}$ plane and which value is also given in Ref. 14 . The effect of the $Q^{2}$ evolution on the ratio $H_{f}^{2 h} / D_{f}^{2 h}$ has been evaluated to be less than $10 \%$ in Ref. 15 for the two experiments and the quantities of Eq. (4) and (6) can be replaced by the corresponding ones calculated from the BELLE results. The ratios $\frac{I}{C}(z)=A_{p}^{2 \pi}(z) / \beta(z)$ and $\frac{I}{C}\left(M_{i n v}\right)=A_{p}^{2 \pi}\left(M_{i n v}\right) / \beta^{\prime}\left(M_{i n v}\right)$ calculated in this way are shown in Fig. 4. As expected they do not show a depepndence on $z$ or $M_{i n v}$. Thus the ratio $I / C$ can be extracted by fitting with a constant the values $\frac{I}{C}(z)$, picture at the left in Fig.4, or the values $\frac{I}{C}\left(M_{i n v}\right)$, picture at the right in Fig.4. The results obtained are: $0.061 \pm 0.009$ and $0.050 \pm 0.008$ from the fits to $\frac{I}{C}(z)$ and to $\frac{I}{C}\left(M_{i n v}\right)$ respectively. Only the statistical errors are taken into account. These are very preliminary results and the evaluation of the final systematic error is still ongoing. It will be interesting to compare our measured value of $I / C$ with the one expected from Eq. (2) and (3) when using known values of $f_{1}^{q}$ and the available parametrizations for the transversity function. Moreover results on the di-hadron asymmetries on polarized deuteron will allow to measure the integral of $h_{1}^{u-\bar{u}}(x)$ and $h_{1}^{d-\bar{d}}$ separately.

\section{References}

1. COMPASS Collaboration (C. Adolph et al.), Phys. Lett. B736, 124 (2014)

2. X. Artru and J. C. Collins, Eur. Phys. J. C69, 277 (1996)

3. A. Bacchetta and M. Radici, Phys. Rev. D67, 094002 (2003)

4. C. Braun for the COMPASS Collaboration, COMPASS results on the transverse spin asymmetry in identified di-hadron production in SIDIS, in Proc. of XXII Int. Workshop on Deep-Inelastic Scattering and Related Subjects, PoS 203 (2014)

5. COMPASS Collaboration (M. Alekseev et al.), Phys. Lett. B673, 127 (2009)

6. COMPASS Collab. (C. Adolph et al.), Phys. Lett. B 713, 10 (2012).

7. A. Bacchetta and M. Radici, Phys. Rev. D74, 114007 (2006)

8. J. She, Y. Huang, V. Barone, B.-Q. Ma, Phys. Rev. D77, 014035 (2008)

9. COMPASS Collab. (C. Adolph et al.), Phys. Lett. B 736, 124 (2014).

10. A. Bacchetta, A. Courtoy and M. Radici, Phys. Rev. Lett. 107, 012001 (2011)

11. C. Elia, Measurement of two-hadron transverse spin asymmetries in SIDIS at COMPASS, PhD Thesis, University of Trieste, 2012

12. A. Bacchetta, A. Courtoy and M. Radici, JHEP 1303, 119 (2013).

13. M. Anselmino, M. Boglione, U. D'Alesio, S. Melis, F. Murgia and A. Prokudin, Phys. Rev. D87, 094019 (2013)

14. BELlE Collab. (A. Vossen et al.), Phys. Rev. Lett. 107, 072004 (2011)

15. A. Courtoy, A. Bacchetta, M. Radici and A. Bianconi, Phys. Rev. D85, 114023 (2012) 